\title{
Use of Virtual Reality in designing urban furniture
}

\author{
Hristiyan Toshkov Petrov ${ }^{1}$ \\ 1 - Technical University of Varna, Department of Industrial Design, 9010, 1 Studentska Street, Varna, Bulgaria \\ Corresponding author contact: hpetrovegmail.com
}

\begin{abstract}
Virtual Reality does not have to be limited to only being a representation and experiential tool - it can be a powerful way of conceiving objects for our physical world as well. The unique features of this technology revolve around the usage of peripheral devices such as controllers (joysticks) and gloves in tandem with VR goggles. By using this type of modeling, designers can overcome some of the current limitations of the design process such as transitioning from sketch to model, scalability of physical models and manipulation difficulties of computer generated models.

An analysis has been made of the evolution of design methodology and its natural progression to virtual and augmented reality. Traditionally the design process of urban design objects starts on paper is evolved through computer modeling and is later tested via physical models and full-scale prototypes. Virtual reality modeling can significantly optimize this process by merging several of the design development phases into one. Sketching, building and testing can be done fully in the virtual environment and the representation of newly created objects will no longer be limited to a $2 D$ surface such as a sheet of paper or a computer screen. The transition to $3 D$ printing is also streamlined with the outcome of the VR designed object being a clear manifestation of the object created in virtual reality.

The goal of the study is to develop a piece of urban furniture, using a virtual reality headset, joysticks and modeling software, manipulate its features and multiply/scale it within the digital environment. The research question is whether such modeling can be precise enough to not only be used as a sketching and sculpting tool but can become the next frontier after computer $3 D$ modeling. The experiment is carried out in two different parts of the world simultaneously - USA and Bulgaria and conceived and manipulated in real-time. The results are analyzed and the advantages and disadvantages of the approach are compared to current design development tools.
\end{abstract}

Key words: Design methods, Industrial Design, Urban Furniture, Virtual Reality Modeling

\section{Introduction}

Frequently used terms and abbreviations:

VR - Virtual reality

AR - Augmented reality

MR - Mixed reality

$\mathrm{CAD}$ - Computer aided design

Virtual reality represents the ultimate medium for design conception. Not only are we able to test ideas in full scale and insert them in their surroundings immediately but VR also offers methods and tools for knowledge sharing in product development, across multiple geographic points, in real-time. The paper outlines a linkage between innovation and the available design methods through the years and the natural progression towards VR.

The development of VR is traced from its origins to the newly uncovered potentials, possible now because of technological advancements in processing power and display. These new tools available to designers should be able to unlock new potentials in the design process. The key benefits of VR as a design tool are listed and current challenges are discussed. 
An experiment is prepared to test the VR design ideas, while taking into consideration urban design principles, context and ergonomic requirements. A solar cell phone charging station is then designed by two collaborating designers from two geographically distant points and the result is placed within a previously built 3D model of a site. The newly created object is evaluated for its aesthetic values, ergonomic advantages and level of inscription into its environment.

\section{Evolution of design development techniques}

Design development is nowadays entirely unthinkable without the use of technology. From smartphones, gadgets, cars and buildings to pieces of clothing and even food - everything that we use in our daily lives has either been fully conceived or - to some extent - been in contact with a computer or high-tech manufacturing. However, that was not the case just twenty to twenty-five years ago. Drafting was still done by hand until relatively recently and prototypes in clay or heavy paper were the only way to experience industrial design objects or architecture in three dimensions (Bertol, Foell, 1997).

Historically, the path of design from an idea and mental construct to realization and embodiment has been closely related to the methods of depiction of geometries, through points and lines using graphite on paper. Nowadays computer software and the way humans interact with it define the limits of what is possible to conceive and then manufacture (Antonelli, 2011).

Virtual reality, as a progression of the current computing technology seems like the next logical step in the field of design because not only is it a powerful, immersive representation tool but its final product can also be enhanced by the use of augmented and mixed realities.

\section{Origins of digitization and early experiments with CAD technologies}

The advent of mathematics and geometry into the design process originates with the contribution of Rene Descartes to the field of art and the introduction of analytical geometry, which uses algebraic methods in the description of geometric objects. This process of unification created in the 17th Century really laid out the foundation of modern computer aided design. After meeting with the Dutch philosopher and scientist Isaak Beeckman, who sparked his interest in mathematics and New Physics, Descartes became convinced that the key to providing a solid framework for philosophy, with all its ambiguities and uncertainties is in fact mathematics (http://www.storyofmathematics.com/17th_descartes.html).

In his ground-breaking work published in 1637 called "Discours de la methode", Descartes included an appendix named "La Geometrie", which introduced concepts and notations widely used in mathematics and geometry till now. He also proposed that each point in two-dimensions plane can be described by two numbers, one giving the point's horizontal location and the other the vertical location, which are now known as Cartesian coordinates. He used two perpendicular lines (also called axes), crossing at a point called the origin, to measure the horizontal $(x)$ and vertical $(y)$ locations. Each axis had a positive and negative value, thus effectively dividing the plane up into four quadrants.

Having achieved that, any equation could now be represented on the plane by plotting on it the solution set of the equation. For example, the equation $y=x$ yields a straight line linking together the points $(0,0)$, $(1,1),(2,2),(3,3)$, etc. The equation $y=2 x$ yields a straight line linking together the points $(0,0),(1,2),(2,4)$, $(3,6)$, etc. More complex equations involving $x^{2}, x^{3}$, etc., plot various types of curves on the plane.

As a point moves along a curve, then, its coordinates change, but an equation can be written to describe the change in the value of the coordinates at any point in the figure. Using this novel approach, it soon became 
clear that an equation like $x^{2}+y^{2}=4$, for example, describes a circle; $y^{2}-16 x$ a curve called a parabola; $x^{2} / a^{2}+y^{2} / b^{2}=1$ an ellipse; $x^{2} / a^{2}-y^{2} / b^{2}=1$ a hyperbola; etc. (Miheli, Novak, Begus, 2014).

Descartes' work, usually referred to as analytic geometry or Cartesian geometry, had the effect of allowing the conversion of geometry into algebra (and vice versa). Thus, a pair of simultaneous equations could now be solved either algebraically or graphically (at the intersection of two lines). It allowed the development of Newton's and Leibniz's subsequent discoveries of calculus. It also unlocked the possibility of navigating geometries of higher dimensions, impossible to physically visualize - a concept which was to become central to modern technology and physics - thus transforming mathematics forever.

Nowadays, several centuries after Descartes's work, the evolution of design development relies entirely on the relationship between algebra and geometry using computer software aimed at the design industry. Programs like AutoCAD, Revit, Maya, 3D Studio Max and Rhino all use mathematical calculations to locate and extrapolate points on two and three-dimensional grids, which are then used to create physical objects via traditional construction methods or 3D printing. The origin of these technological advancements can be traced to a group of MIT researchers who gathered at the beginning of 1960s to develop computer graphics aimed specifically at the design industry (Rix, Haas, Teixeira, 2016). Their work of course would not be possible without the substantial advancements in computational power and the development of computer screens around the same time period.

One of the key developments in the computer graphic field was made by Ivan Sutherland in his $\mathrm{PhD}$ thesis called "Sketchpad: a Man-Machine Graphical Communication System", 1962 (Fig.1). Considered by many a foundational work for modern computer graphics, the program uses drawing as a novel communication medium for the computer (Schodek, Bechtold, Griggs, Kao, Steinberg, 2005). A Sketchpad user would sketch directly on a computer display with a "light pen." The light pen was used both to position parts of the drawing on the display and to point to them in order to change them. Sketchpad was a general purpose system and has been used to draw electrical, mechanical, scientific, mathematical, and animated drawings. The program also made it easy to draw highly repetitive or highly accurate drawings, and to modify elements previously drawn with it with ease - something very laborious until then, as it was performed on paper.

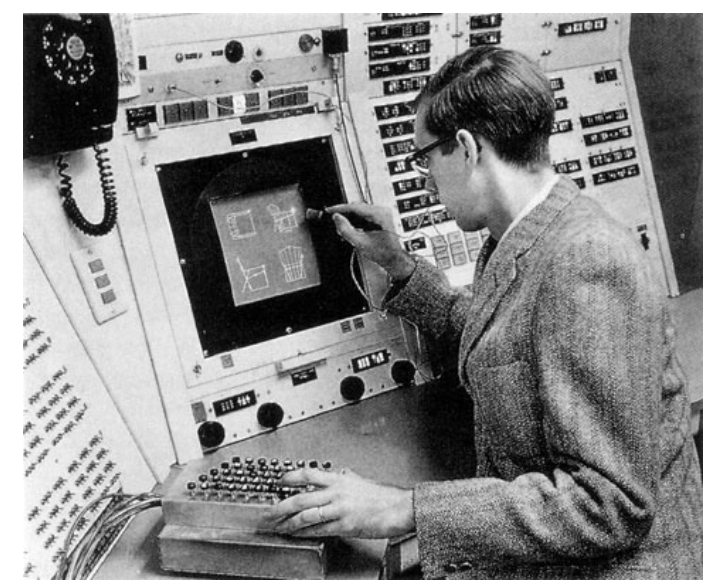

Figure 1: Ivan Sutherland using Sketchpad in 1962

Later on, in the 1960s, the French engineer Pierre Bezier deploys several mathematical principles earlier developed by Sergei Bernstein (1912) and Paul de Casteljau (1959) to analyze and describe complex curves used in the automotive industry. While working for French automaker Renault, Bezier widely used and publicized these parametric curves, based on Bernstein polynomials (an expression consisting of variables and coefficients, that involves only the operations of addition, subtraction, multiplication and non- negative integer exponents of variables, e.g. $\left.x^{2}-4 x+7\right)$ in order to manufacture double-curvature surfaces and these curves later on started bearing his name. The underlying principles of the Bezier curves in 
tandem with NURBS curves are currently widely used in 3D software to design and describe complex shapes and surfaces.

\section{Early VR development}

Virtual reality as we know it today has come about as a result of the progress in several technological fields - computer games, military industry and high-tech manufacturing but just as any other paradigm shift, it takes time for it to get fully established. The term has entered our social culture permanently and thanks to movies and science fiction novels most people have at least some understanding of it.

One of the pioneers of virtual reality was the cinematographer Morton Heilig (Jerald, 2016). In 1957 he built and patented "Sensorama" (Fig.2) - a machine which simulated cycling within an urban environment. The person using would take a seat and put their head in front of a display which showed moving city-scape images along with sounds and scents from the streets. "Sensorama" was the first step in the development of virtual reality and although the machine was not a market success, it laid the foundation for immersive experience technology.

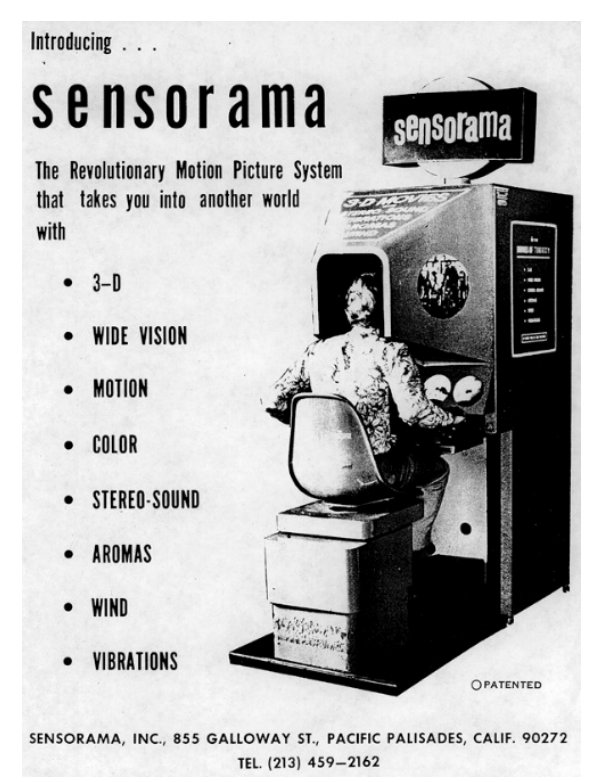

Figure 2: Sensorama introduced a new type of olfactory experience in the 1950s

Following an influential paper published in 1965 and called "The Ultimate Display" in which he predicted the modern rise of virtual reality, Ivan Sutherland alongside his student Bob Sproull developed the first ever head-mounted augmented reality display at the University of Utah. Produced in 1968, it was designed to help helicopter pilots land their machines safely at night. The technology was revolutionary for its time as it used head and eye tracking and was the first to blend reality with virtual reality - the users wore clear lenses and on which an image of a spatial wireframe was projected, thus enhancing reality to become an augmented reality device. Because the machine required multiple elements and was suspended from the ceiling it was called "The Sword of Damocles" (Fig.3) (Armstrong, 2016). 


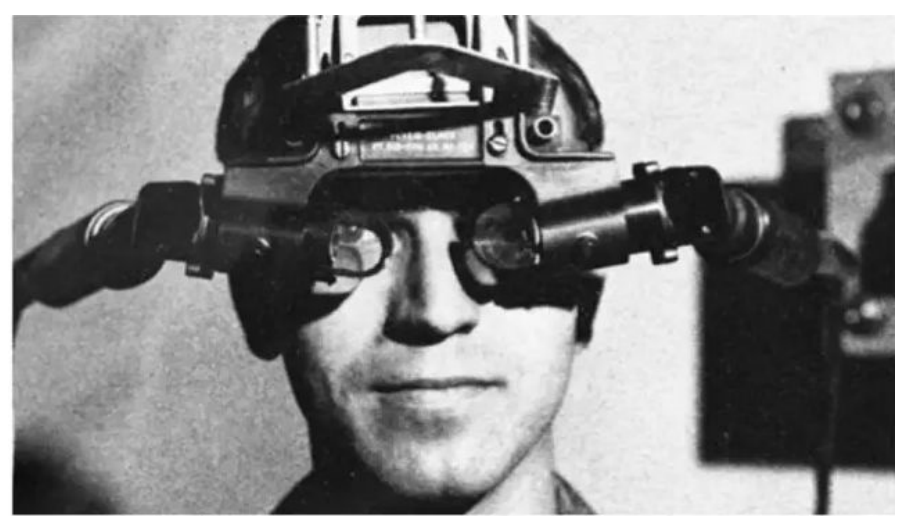

Figure 3: Ivan Sutherland: Sword of Damocles 1968

\section{Using VR as a design generative tool}

Thanks to recent advancements in processing power virtual reality is gradually revealing its potential as an appealing generative tool for the design industry. Currently, there are several software platforms that have been developed in the field primarily for the gaming and film industries but the potential of applying these in the creation of industrial design objects is latent and needs to be explored further. There are several key benefit to using this technology is the ability for direct input using motion-tracking joysticks with embedded haptics.

\subsection{Key disadvantages associated to current digital modeling:}

5.1.1 Detached input: Using a mouse on a 2D computer screen which uses "simulated" perspective and three-dimensionality is time-consuming and skill and knowledge demanding to conceive and design objects.

5.1.2 Lack of full integration: It still necessary to build a physical mock-up of any industrial design object to fully understand scale, fitting and usability.

\subsection{Key benefits to VR design:}

5.2.1 Direct input: While the computer mouse is a great tool for current computer screens, when we are working directly in 3D space, it is no longer sufficient to use a 2D device. Through the incorporation of joysticks, we can use our hands directly in the creation process. The multi-axial rotation of the human hand is still unrivaled by any machine and can in this case facilitate the conception of highly detailed products, which should be able to address a larger set of issues than current design methods.

5.2.2 Immersive experience: When we are creating directly in 3D, our bodies are inherently part of the process of conception and can be used directly as subjects or objects of the design. Issues of ergonomics can be addressed directly in the design process, which of course allows for better fitting and more detailed final products than the ones we use today. Moreover, through the implementation of 3D printers at the end of the VR design process, we can achieve mass customization as well - for example frames for prescription glasses can be designed directly on people's faces for a more comfortable fit or everyday objects such as cutlery and cups can be made to fit precisely into the user's hands. 
5.2.3 Direct application: By using an augmented reality (AR) headset or after syncing a laser 3D scan with a VR headset, we can quickly insert the newly created objects within their environment, scale and multiply them in the field and really get a sense of the final outcome of the design and how they sits in their surroundings.

5.2.4 Integration of product development phases: From sketching, scaling, material testing and prototyping - all can be done in the virtual environment without having to build costly mock-ups, thus ensuring cost effectiveness (Bordegoni, Rizzi, 2011).

5.2.5 Real-time modification of existing objects: When we have already transferred the 3D information of an existing environment or an object to VR, we can make modifications to it in real-time by changing their shape, surfaces or color and then "insert" it back in its surrounding.

5.2.5 Remote collaboration: Designers no longer have to occupy the same space in order to collaborate on creative projects. Just by using a headset and Internet connection, a designer in Tokyo can work alongside a colleague in New York while actively manipulating the same object in real time. Moreover, the collaboration does not need to be limited to designers developing an idea - consultants, officials and regulators can also "chime-in" during the design process and ensure that new product meets all norms and regulations.

\subsection{Currently available VR modeling software:}

Oculus Medium: Virtual reality sculpting tool with the most advanced set of tools and focused entirely around the creation of VR objects (Fig.4). The program has been gaining traction with character developers for the gaming industry. Medium is used on the Oculus Rift and uses Oculus Touch controllers. The headset device company, Oculus VR is currently owned and operated by Facebook.

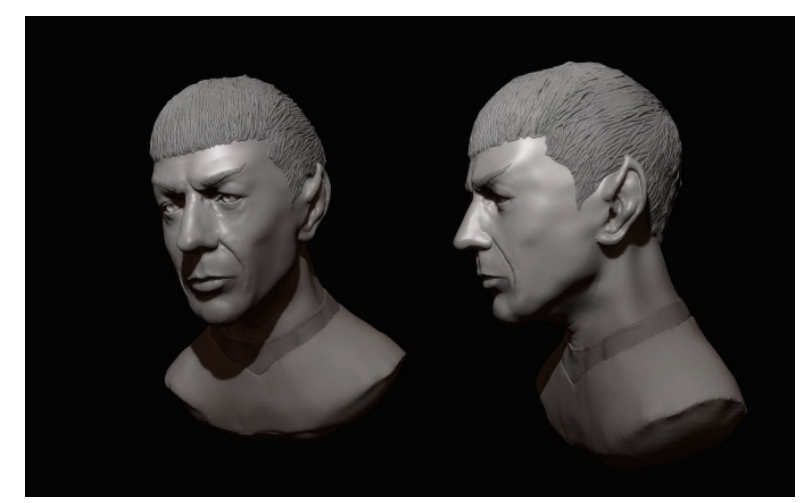

Figure 4: Spock (Star Trek) created using Oculus Rift headset, Touch and Oculus Medium

Tilt Brush: Developed by Google, it allows users to paint in 3D space with a variety of brushes available. Intended for use with the HTC Vive and Oculus Rift. Tilt Brush (Fig.5), as its title suggests has the potential to revolutionize a field considered by many stagnant in the past decades - painting. By adding a third dimension and creating an immersive experience, viewers can now experience a work of art by being in it and seeing it from all possible angles. The program does well with surface oriented objects such as the ones in fashion and industrial design. 


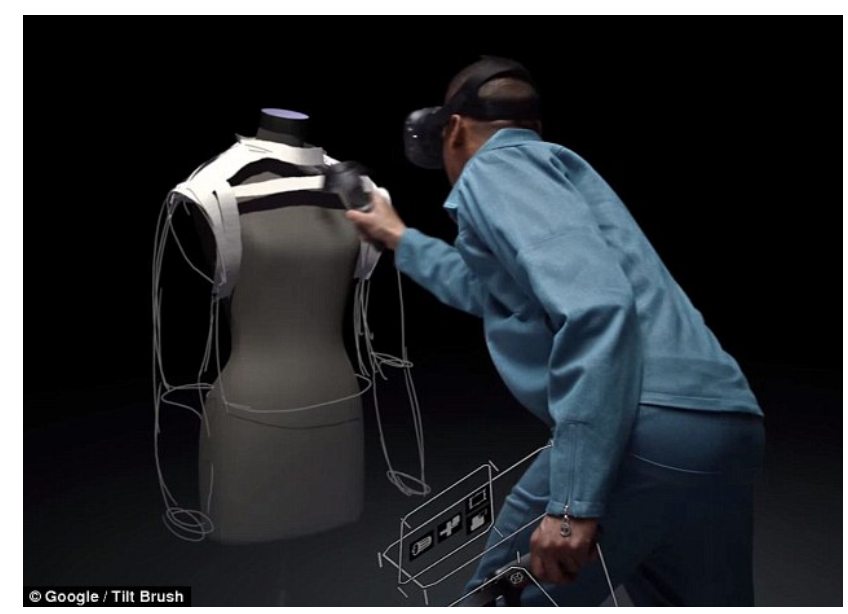

Figure 5: Tilt Brush used to create new clothing designs

Gravity Sketch VR: allows for free-hand drawing, but also has additional tools that lets surfaces and symmetry be manipulated, as well as control-point editing. It can be used with Oculus Rift and HTC Vive. Models created in the program can be exported and further developed in other software platforms with more powerful rendering capabilities for example (Fig.6).

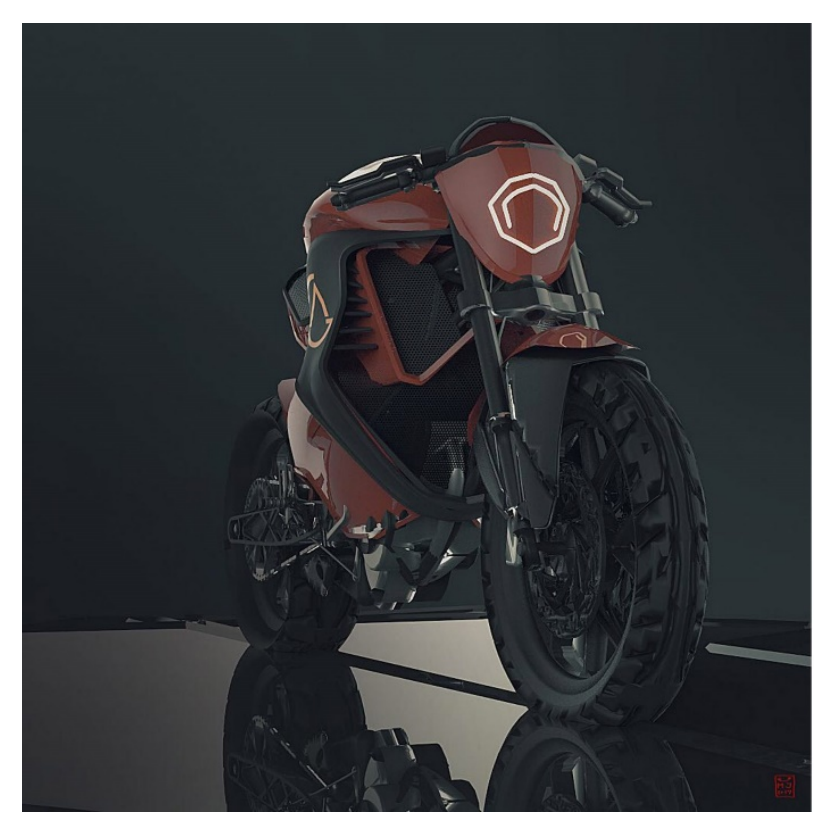

Figure 6: Motorcycle design produced in Gravity Sketch VR

Seymourpowell VR Design Tool: The software tool allows designers and consultants to "dial-in" onto product development sessions and input comments to meet requirements or develop design ideas. In addition to its collaborative functionality, the software features advanced sketching tools, allowing designers to draw smooth curves over set points and work within safety parameters (Fig.7) (https://www.dezeen.com). 


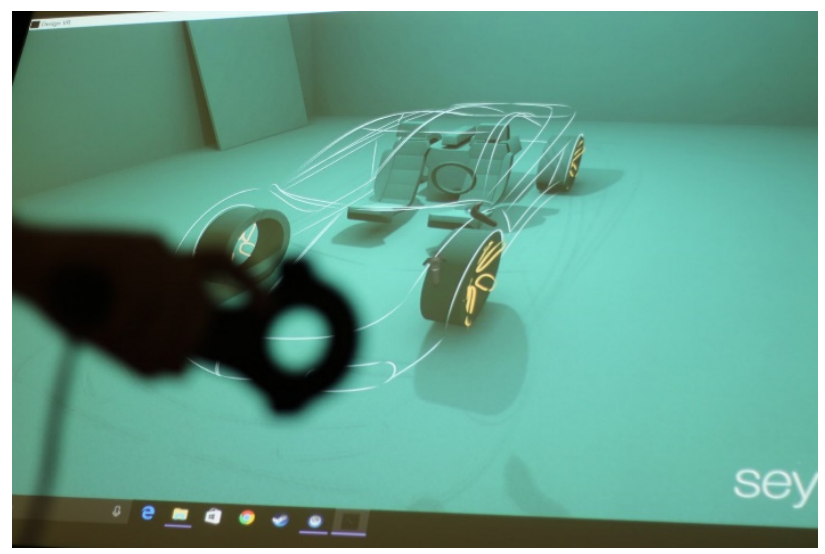

Figure 7: Seymour Powell's VR Design Tool

\subsection{Key challenges in using VR's current design tools}

5.3.1 Precision: Because of the relatively early developmental stage of the software interface devices (controllers/joysticks), the precision we are used to achieving easily with current CAD programs is not yet available with VR tools. The VR sculpting software is capable of achieving definition and "character lines" as well as giving intricate detail to objects - but those cannot be accurately measured. Most VR design tools are still in free-form mode and models need to be exported to CAD programs for measurements.

5.3.2 Cable tethering: Modeling in VR requires a large amount of data processing and in order to achieve a realistic, immersive environment cables are needed to feed image information. With the advancement of processing power and data transferring devices, we should soon be able to use VR devices wirelessly and walk around larger spaces with them.

\section{Virtual reality, augmented reality and mixed reality in the context of design}

It is important to give definitions of virtual reality, augmented and mixed reality in the context of direct design development for the field of industrial design.

Virtual reality: The technology uses a fully immersive headset (HTC Vive, Oculus Rift) to render a computer-generated model of a real or imaginary world which can be explored through physical walking or head/eyes turning. An industrial design object conceived in such environment may or may not follow the rules of real-world applications and the final product may serve a function for the virtual world only or through the incorporation of physical parameters and material properties - may be, later on, built and used in real-world applications. Most sculpting software is currently geared towards VR.

Augmented reality: The term refers to a live view of a physical environment, which is enhanced by a layer of information that is not fixed to an object. The effect is achieved by wearing a transparent set of glasses (Google Glass, Vuzix Blade) with a small projector attached to them. We can get information about the source of the material used in a design, its cost and other important features, just by looking at it. (http://www.vrhuman.com/).

Mixed reality: The technology is associated with a view of the physical world, enhanced by a layer of new information that is fixed to an object. Using mixed reality glasses (Microsoft HoloLens) we can, for example, see what a city bus stop will look like with a digital advertising screen or a transportation schedule screen, which informs passengers of the estimated arrival of a bus. Similarly, we can test different materials on an 
existing real-life object or see a section through a bridge or the cable and pipe system underneath a street or a sidewalk.

\section{Application of VR in the design of urban objects}

Using current Lidar and laser scanning technologies (Whyte, 2002), we can re-create a comprehensive image of the environment surrounding the urban design object we are about to design. Next, we can insert that information into the VR environment and start designing directly "on site". Because scalability of VR objects is done so easily and quickly, we can manipulate an urban bench for example, form it precisely around a desired body position and then insert in the environment. Created in direct relationship to the body and not on a detached computer screen, the bench will have a much finer relationship to the body positions and will thus be more comfortable and ergonomically fitting for the users. We will also be able to see the design directly in its context and assess easily whether or not it is a good fit.

Similarly, the design of a street light for example can be done in VR and instead of running complex photon calculations on specialized software to predict light distribution on the street below, we can test different geometries of the post, different heights and distances from the ground and one-another, multiply them in perspective and experience the result in real time. Standardization of produced results will be measured against the guidelines as described by the Urban Design Standards (A.P.A, Steiner, Butler, 2006)

Urban bike stands for example can also be designed/sketched directly in VR. The structure can be formed around an actual bicycle, or several of them, and the newly created object can be immediately "placed" in its environment for city officials to approve. The same holds true for water fountains, which can be assessed immediately as well as photovoltaic phone charging stations, digital announcement boards or public transport shelters - all of which can be designed around the people who will be using them.

\section{VR experiment to be carried out}

Two designers will be asked to deploy VR headsets and collaborate on the design of an urban design piece. The context or the surrounding area of the project will be provided either through laser scanning or conventional 3D modeling. The task will be to design a solar charging cell phone station which doubles its function as a public seating arrangement as well. The aesthetic, ergonomic and urban qualities of the newly designed object are assessed against a set of criteria used to approve new proposals.

\section{Conclusion}

VR with its immersive and inherently spatial qualities seems like an excellent tool for the industrial design field with the potential of being its the next frontier. It currently has some challenges to be overcome but with input from designers and some fine-tuning and development of current software, we can see a move from the computer screen/mouse to the VR goggles/controllers set up as a stage for design development.

Although it is too early to draw conclusions, as the experiment for this study is yet to be carried out, the goal is to produce novel designs, which are in better sync with their environment, more ergonomically successful and overall more thoroughly thought out. Because of the direct input into the design process and the freedom from limitations imposed by current 3D programs, the outcome should have a recognizable and unique appearance as well. 


\section{References}

American Planning Association (2006). Planning and Urban Design Standards. John Wiley and Sons

Antonelli, Paola (2011). Talk to me: Design and the communication between People and Objects. The Museum of Modern Art, New York

Armstrong, Helen - edited. (2016). Digital Design Theory: Readings from the Field. Chronicle Books

Bertol, Daniela \& Foell, David (1997). Designing Digital Space: An Architect's Guide to Virtual Reality.

Bordegoni, Monica \& Rizzi, Caterina (2011). Innovation in Product Design: From CAD to Virtual Prototyping. Springer Science \& Business Media

Carmona, Matthey (2010). Public Places, Urban Spaces: The Dimensions of Urban Design. Routledge

Jerald, Jason (2016). The VR Book: Human-Centered Design for Virtual Reality. ACM Books

Hurst, Ken (1999). Engineering Design Principles. Butterworth-Heinemann

Martegani, Paolo \& Montenegro, Ricardo (2000). Digital Design: New Frontiers for the Objects. Springer Science \& Business Media

Mihelj, Matjaz \& Novak, Domen \& Begus, Samo (2013). Virtual Reality Technology and Applications, Volume 67. Springer Science \& Business Media

Penichet, Victor \& Penalver, Antonio \& Gallud, Jose (2013). New Trends in Interaction, Virtual Reality and Modeling. Springer

Schodek, Daniel \& Bechthold, Martin \& Griggs, Kimo \& Martin Kao, Kenneth \& Steinberg, Marco (2005). Digital Design and Manufacturing. John Wiley and Sons

Rix, J. \& Haas, S. \& Teixeira, J. (2016). Virtual Prototyping: Virtual Environments and the product design process. Springer.

Whyte, Jennifer (2002). Virtual Reality and the Built Environment. Routledge

\section{Internet Sources}

http://www.storyofmathematics.com/17th_descartes.html

https://www.dezeen.com

http://www.vr-human.com/ 